\title{
On the truncation of the harmonic oscillator wavepacket
}

\author{
L. Rebollo-Neira and S. Jain \\ Aston University, \\ Birmingham B4 7ET, United Kingdom
}

\begin{abstract}
We present an interesting result regarding the implication of truncating the wavepacket of the harmonic oscillator. We show that disregarding the non-significant tails of a function which is the superposition of eigenfunctions of the harmonic oscillator has a remarkable consequence. Namely, there exit infinitely many different superpositions giving rise to the same function on the interval. Uniqueness, in the case of a wavepacket, is restored by a postulate of quantum mechanics.

PACS: 03.65.-w, 03.65.Ca
\end{abstract}

\section{Introduction}

We analyse the effect of truncating the wavepacket of the harmonic oscillator in the light of the frame theory. Such a theory, developed in 1952 by Duffin and Shaffer in the context of harmonic analysis [1] has been applied, for over fifteen years, to construct coherent states. More specifically, we should mention affine coherent states, also called wavelets, and Weyl-Heisenberg coherent states, also known as Gabor frames [2-11]. We recall in the next paragraph the general definition of frames and a few properties, which is all what we need for the purpose of the present effort. For a complete treatment of frames we refer to [12-14].

Given a Hilbert space $\mathcal{H}$, a family $\left\{\phi_{n}\right\}_{n \in \mathbb{N}}$ in $\mathcal{H}$ is called a frame for $\mathcal{H}$ if for every $f \in \mathcal{H}$ there exists a pair of constants $0<A \leq B<\infty$ such that

$$
A\langle f, f\rangle \leq \sum_{n \in \mathbb{N}}\left|\left\langle\phi_{n}, f\right\rangle\right|^{2} \leq B\langle f, f\rangle .
$$

The constants $A$ and $B$ are called the frame bounds and (1) the frame condition. From its definition it is clear that a frame is a complete set, since the relations $\left\langle\phi_{n}, f\right\rangle=0, n \in \mathbb{N}$ imply 
that $f \equiv 0$. The removal of an element from a frame leaves either a frame or an incomplete set. A frame that ceases to be complete if an arbitrary element $\phi_{n}$ is removed, is called exact. This last property implies that only exact frames are bases; in the general case the family $\left\{\phi_{n}\right\}_{n \in \mathbb{N}}$ may be over complete. When the condition $A=B$ holds, the frame is said to be a tight frame. Assuming that all the elements $\phi_{n}, n \in \mathbb{N}$ are normalised to unity, a tight frame is an orthogonal basis if and only if $A=B=1$. Notice that this implies that a tight frame with frame bounds $A=B=1$ is an orthogonal basis only if the elements are normalised to unity, otherwise it is a redundant frame.

In this paper we introduce a class of redundant tight frames which are trivially obtained by redefining the functions of an orthonormal basis to be zero outside an interval. Such a restriction allows us to use the truncated functions to represent a given function vanishing outside the identical interval. This has a remarkable consequence, namely, the coefficients of the corresponding linear span are not unique. We discuss this important consequence in relation to the truncation of the wavepacket of an harmonic oscillator. We show that, by disregarding the non-significant tails of a function which is the superposition of the harmonic oscillator eigenfunctions one creates a null space. As a consequence, the restriction of the wavepacket to the reduced interval can be realized by infinitely many different coefficients giving rise to the same function on the interval. This is, certainly, a striking result. Nevertheless, uniqueness can be restored by means of one of the postulates of quantum mechanics.

The paper is organised as follows: In Section II we introduce the construction of tight frames for the Hilbert space of functions vanishing outside an interval by simple truncation of orthonormal basis functions. In Section III we apply these results to analyse the effect of truncating the wavepacket of the harmonic oscillator. The conclusions are drawn in Section IV. 


\section{Building tight frames from orthonormal bases}

The next proposition shows that, for the space of square integrable functions vanishing outside an interval, one can construct tight frames by simple restriction of orthonormal functions to the corresponding interval.

Proposition 1. Let $\left\{\psi_{n}\right\}_{n \in \mathbb{N}}$ be an orthonormal basis for $L^{2}\left[L_{1}, L_{2}\right]$, i.e.,

$$
\left\langle\psi_{m}, \psi_{n}\right\rangle=\int_{L_{1}}^{L_{2}} \psi_{m}^{*}(x) \psi_{n}(x) d x=\delta_{m, n}
$$

and $\chi_{\left[L_{1}^{\prime}, L_{2}^{\prime}\right]}$ the characteristic function for the interval $\left[L_{1}^{\prime}, L_{2}^{\prime}\right]$, i.e,

$$
\chi_{\left[L_{1}^{\prime}, L_{2}^{\prime}\right]}(x)= \begin{cases}1 & \text { if } x \in\left[L_{1}^{\prime}, L_{2}^{\prime}\right] \\ 0 & \text { otherwise. }\end{cases}
$$

Functions $\left\{\psi_{n}^{\prime}\right\}_{n \in \mathbb{N}}$, obtained as $\psi_{n}^{\prime}(x)=\chi_{\left[L_{1}^{\prime}, L_{2}^{\prime}\right]}(x) \psi_{n}(x), n \in \mathbb{N}$, with $\left[L_{1}^{\prime}, L_{2}^{\prime}\right] \subset\left[L_{1}, L_{2}\right]$, constitute a tight frame for the subspace $\mathcal{W}$ of square integrable functions vanishing outside $\left[L_{1}^{\prime}, L_{2}^{\prime}\right]$

Proof. On the one hand, since $\left\{\psi_{n}\right\}_{n \in \mathbb{N}}$ is an orthonormal basis of $L^{2}\left[L_{1}, L_{2}\right]$, for all $f \in$ $L^{2}\left[L_{1}, L_{2}\right]$ we have

$$
\|f\|^{2}=\langle f, f\rangle=\sum_{n \in \mathbb{N}}\left\langle f, \psi_{n}\right\rangle\left\langle\psi_{n}, f\right\rangle .
$$

On the other hand, for all $f \in \mathcal{W}$ it is true that $\chi_{\left[L_{1}^{\prime}, L_{2}^{\prime}\right]} f=f$ and we further have:

$$
\sum_{n \in \mathbb{N}}\left\langle f, \psi_{n}^{\prime}\right\rangle\left\langle\psi_{n}^{\prime}, f\right\rangle=\sum_{n \in \mathbb{N}}\left\langle f \chi_{\left[L_{1}^{\prime}, L_{2}^{\prime}\right]}, \psi_{n}\right\rangle\left\langle\psi_{n}, \chi_{\left[L_{1}^{\prime}, L_{2}^{\prime}\right]} f\right\rangle=\|f\|^{2}
$$

which proves that $\left\{\psi_{n}\right\}_{n \in \mathbb{N}}$ is a redundant tight frame for $\mathcal{W}$, since the elements $\psi_{n}^{\prime}$ are not normalised to unity.

We prove next that, through the finite subset of frames elements $\psi_{1}^{\prime}, \ldots, \psi_{N}^{\prime}$ constructed as indicated in Proposition 1 we can construct the orthogonal projection of $f$ onto $\mathcal{S}=$ $\operatorname{span}\left\{\psi_{1}, \ldots, \psi_{N}\right\}$, restricted to $\left[L_{1}^{\prime}, L_{2}^{\prime}\right]$. 
Proposition 2. Let $\psi_{1}^{\prime}, \ldots, \psi_{N}^{\prime}$ be as defined in Proposition $\mathbf{1}$, and for each $f \in \mathcal{W}$ let us define a function $f^{N}$ as:

$$
f^{N}=\sum_{i=1}^{N} \psi_{i}^{\prime}\left\langle\psi_{i}^{\prime}, f\right\rangle
$$

The function given in (41) satisfies:

$$
f^{N}=\left\{\begin{array}{cl}
\hat{P}_{\mathcal{S}} f(x) & \text { if } x \in\left[L_{1}^{\prime}, L_{2}^{\prime}\right] \\
0 & \text { otherwise }
\end{array}\right.
$$

where $\hat{P}_{\mathcal{S}}$ stands for the orthogonal projector operator onto $\mathcal{S}$.

Proof. Since $\left\{\psi_{1}, \ldots, \psi_{N}\right\}$ is an orthonormal set for $L^{2}\left[L_{1}, L_{2}\right]$, the orthogonal projection of $f \in L^{2}\left[L_{1}, L_{2}\right]$ onto $\mathcal{S}$ is given as

$$
\hat{P}_{\mathcal{S}} f=\sum_{i=1}^{N} \psi_{i}\left\langle\psi_{i}, f\right\rangle
$$

For $f \in \mathcal{W}$ it holds that

$$
\sum_{i=1}^{N} \psi_{i}\left\langle\psi_{i}, f\right\rangle=\sum_{i=1}^{N} \psi_{i}\left\langle\psi_{i}^{\prime}, f\right\rangle
$$

Then

$$
\hat{P}_{\mathcal{S}} f=\sum_{i=1}^{N} \psi_{i}\left\langle\psi_{i}^{\prime}, f\right\rangle
$$

and the proof follows by multiplication of both sides of the equation by $\chi_{\left[L_{1}^{\prime}, L_{2}^{\prime}\right]}$.

A convenient property of tight frames is that the coefficients of a linear span in a tight frame superposition are obtained by inner products with the frame functions. In our context this implies that the coefficients of an orthonormal expansion and the ones to span a function in $\mathcal{W}$ by means of the frame in Proposition 1 are computed in an equivalent manner. The essential difference is that, as discussed below, the coefficients in the frame superposition are not unique.

Let us consider the frame constructed in Proposition 1 and let us define $\mathcal{S}^{\prime}=\operatorname{span}\left\{\psi_{1}^{\prime}, \ldots, \psi_{N}^{\prime}\right\}$. For every $f \in \mathcal{S}^{\prime} \subset \mathcal{W}$ we have:

$$
f=\sum_{n=1}^{N} c_{n} \psi_{n}^{\prime}, \quad \text { with } \quad c_{n}=\left\langle\psi_{n}^{\prime}, f\right\rangle
$$


Since $f \in \mathcal{W}$ it is true that $c_{n}=\left\langle\psi_{n}^{\prime}, f\right\rangle=\left\langle\psi_{n}, f\right\rangle$ and the equivalence with the orthonormal case follows. However, the redundancy of the frame implies that the coefficients in (7) are not unique. Indeed, since a redundant frame is linearly dependent, the following situation can occur:

$$
0=\sum_{n=1}^{N} c_{n}^{\prime} \psi_{n}^{\prime}, \quad \text { for } \quad \sum_{n=1}^{N}\left|c_{n}^{\prime}\right|^{2} \neq 0 .
$$

Taking inner product both sides of the equation on the left with each $\psi_{m}^{\prime}$ we have

$$
0=\sum_{n=1}^{N} c_{n}^{\prime}\left\langle\psi_{m}^{\prime}, \psi_{n}^{\prime}\right\rangle
$$

that we can recast as

$$
0=G \overrightarrow{c^{\prime}}
$$

where $G$ is a matrix of elements $g_{m, n}=\left\langle\psi_{m}^{\prime}, \psi_{n}^{\prime}\right\rangle, n, m=1, \ldots, N$ and $\overrightarrow{c^{\prime}}$ a vector, the component of which are the coefficients $c_{n}^{\prime}, n=1, \ldots, N$. Equations (8) and (10) imply that the general form for (17) is

$$
f=\sum_{n=1}^{N} c_{n} \psi_{n}^{\prime}+\sum_{n=1}^{N} c_{n}^{\prime} \psi_{n}^{\prime}
$$

with $c_{n}, n=1, \ldots, N$ given in (17) and $c_{n}^{\prime}, n=1, \ldots, N$ the components of a vector $\overrightarrow{c^{\prime}} \in \operatorname{null}(G)$.

It is appropriate to stress the significance of Proposition 1$]$ when the interval $\left[L_{1}, L_{2}\right]$ is actually the whole real line. Then, for numerical calculations one is obliged to work on a finite domain. An important consequence of this fact will be discussed in the next section.

\section{On the truncation of the harmonic Oscillator wavepacket}

We show here that the results of the previous section are relevant to the analysis of the truncation of the wavepacket of the harmonic oscillator. To this end we simulate two different situations, which are specially devised to illustrate the phenomenon we wish to discuss.

Let us consider that a normalised to unity function $\Psi(x) \in \mathcal{H}$ is generated as linear superposition of the harmonic oscillator eigenfunctions, i.e.,

$$
\Psi(x)=\sum_{n=1}^{N} c_{n} \frac{e^{-0.5 x^{2}} H_{n}(x)}{\sqrt{2^{n-1}(n-1) ! \sqrt{\pi}}}, \quad n, m=1, \ldots, N,
$$


where we have written $\psi_{n}(x)=\frac{e^{-0.5 x^{2}} H_{n}(x)}{\sqrt{2^{n-1}(n-1) ! \sqrt{\pi}}}$ in terms of the Hermite polynomial $H_{n}(x)$. The coefficients $c_{n}, n=1, \ldots, N$ in (12) are simulated according to the equation:

$$
c_{n}=\frac{e^{-0.0032(n-80)^{2}}}{\sqrt{\sum_{n=1}^{N} e^{-0.0064(n-80)^{2}}}} .
$$

We consider $N=160$. The left graph of Figure 1 depicts the corresponding function for $x \in[-1,30]$. Since the values $\Psi(x)$ are very small outside this interval $\left(\Psi(-1)=8.7 \times 10^{-11}\right.$ and $\left.\Psi(30)=2.7 \times 10^{-92}\right)$ one does not commit a significant error by calculating expectation values using this domain. In order to illustrate this fact let us calculate $\bar{x}$ and $\overline{x^{2}}$ as

$$
\begin{aligned}
\bar{x} & =\int_{-40}^{40}|\Psi(x)|^{2} x d x=12.56967570231863 \\
\bar{x} & =\int_{-1}^{30}|\Psi(x)|^{2} x d x=12.56967570231863 \\
\overline{x^{2}} & =\int_{-40}^{40}|\Psi(x)|^{2} x^{2} d x=158.4912423027778 \\
\overline{x^{2}} & =\int_{-1}^{30}|\Psi(x)|^{2} x^{2} d x=158.4912423027777 .
\end{aligned}
$$

The difference in the values of $\bar{x}$ cannot be observed in the given format and the values of $\overline{x^{2}}$ differ only in the last of the 16 digits. Thus one could conclude that, for the purpose of computing expectation values, neglecting the tails of the distribution outside the interval $x \in[-1,30]$ is not harmful. However, the assumption that $|\Psi(x)|=0$ for $x \notin[-1,30]$ has a tremendous consequence: the coefficients of the superposition (12) are thereby not unique. In order to illustrate this we compute the vectors in the null space of matrix $G$ of elements

$$
g_{m, n}=\int_{-1}^{30} H_{m}(x) H_{n}(x) \frac{e^{-x^{2}}}{\sqrt{2^{m-1}(m-1) ! 2^{n-1}(n-1) !} \pi} d x .
$$

We use just one of the eigenvectors spanning null $(G)$, say the vector $\overrightarrow{c^{\prime}}$, to construct the coefficients $c_{n}^{\prime \prime}=c_{n}+c_{n}^{\prime}, n=1, \ldots, 160$, with $c_{n}$ as in (13) and $c_{n}^{\prime}, n=1, \ldots, 160$ the components of $\overrightarrow{c^{\prime}} \in \operatorname{null}(G)$. The right graph in Figure 1 plots the coefficients $c_{n}^{\prime \prime}$. We use now these coefficients to construct the function $\Psi^{\prime \prime}(x)$. By calculating

$$
\left\|\Psi^{\prime \prime}-\Psi\right\|=\sqrt{\int_{-1}^{30}\left|\Psi^{\prime \prime}(x)-\Psi(x)\right|^{2} d x}=1.4198 \times 10^{-18}
$$


we do not see any significant difference in the functions. However $\left\|\overrightarrow{c^{\prime \prime}}-\vec{c}\right\|=\left\|\overrightarrow{c^{\prime}}\right\|=1$, which clearly shows that the function are considering can be generated in many different ways on the interval $[-1,30]$. Even considering the interval $[-10,40] \operatorname{null}(G)$ is still not empty. It would be empty for $[-15,40]$, though, but only if the maximum number of states $N=160$ is maintained fixed.

It should be noted that, although the 'critical' interval depends on the number $N$ and a smaller value of $N$ would decrease the length of the critical interval, there is room for adjustments. Indeed, by considering zero the coefficients which do not intervene in the original superposition, but allowing the transformation to have larger dimension, the critical interval is enlarged. Notice that this opens the possibility of producing the identical function by means of states that were not present in the original superposition. The next example illustrates this situation.

Consider that the coefficients of the superposition (12) are now simulated as

$$
c_{n}=\frac{e^{-n}}{\sqrt{\sum_{n=1}^{20} e^{-2 n}}}, \quad n=1, \ldots, 20
$$

The corresponding function $\Psi(x)$ is plotted in the left graph of Figure 2. In this case the critical interval yielding lack of uniqueness is included in the main support of $\Psi(x)$. Hence, the restriction to such an interval is not possible. Now, increasing the value of $N$ to 130 for instance, and considering $c_{n}=0, n=31, \ldots, 130$, the function $\Psi(x)$ does not change. Nevertheless, we have a $130 \times 130$ matrix $G$ constructed by extending the interval to one containing the most significant support of $\Psi(x)$ (in this case $[-7,10]$ ). Taking one of the vectors in null $(G)$ we construct the coefficients $\overrightarrow{c^{\prime \prime}}$ by an equivalent process as in the previous example. With this coefficients, plotted in the right graph of Figure 2, we construct the graph on the left of Figure 2. Notice that, the fact that coefficients $c_{n}^{\prime \prime}$ have significant values for $n=31, \ldots, 130$, implies that $\Psi(x)$ can be realized by states which were not present in the original superposition. 
Nevertheless, the average energy is the same. Indeed,

$$
\bar{E}=\left\langle\psi^{\prime \prime}, \hat{H} \psi^{\prime \prime}\right\rangle=\sum_{n=1}^{N} \sum_{m=1}^{N}\left(c_{n}^{*}+c_{n}^{*}\right)\left\langle\psi_{n}^{\prime}, \hat{H}\left(c_{m}+c_{m}^{\prime}\right) \psi_{m}^{\prime}\right\rangle
$$

and, since by hypothesis $\sum_{m=1}^{N} c_{m}^{\prime} \psi_{m}^{\prime},=0$, it follows that

$$
\bar{E}=\sum_{n=1}^{N} \sum_{m=1}^{N} c_{n}^{*}\left\langle\psi_{n}^{\prime}, \hat{H} c_{m} \psi_{m}^{\prime}\right\rangle=\left\langle\psi^{\prime}, \hat{H} \psi^{\prime}\right\rangle .
$$

This result stresses the point that was made initially. Namely, that without normalising the coefficients, there could be infinitely many different ways of realizing the harmonic oscillator wavepacket on a finite interval. However, according to quantum mechanics each $\left|c_{n}^{\prime \prime}\right|^{2}$ represents the probability of finding the harmonic oscillator in the state $n$. Hence, to be able to maintain this interpretation we must impose the normalisation condition on the coefficients $c_{n}^{\prime \prime}$. As a consequence, we cannot use a vector $\overrightarrow{c^{\prime}}$ of arbitrary norm. In fact, in order to construct a normalised vector we need to consider $\overrightarrow{c^{\prime \prime}}=\vec{c}+D \overrightarrow{c^{\prime}}$, with $\overrightarrow{c^{\prime}} \in \operatorname{null}(G)$ and $D$ a constant to be determined by the condition $\left\|\overrightarrow{c^{\prime \prime}}\right\|^{2}=1$. Writing this condition explicitly we have

$$
\left\|\overrightarrow{c^{\prime \prime}}\right\|^{2}=\|\vec{c}\|^{2}+D\left\langle\vec{c}, \overrightarrow{c^{\prime}}\right\rangle+D^{*}\left\langle\overrightarrow{c^{\prime}}, \vec{c}\right\rangle+|D|^{2}\left\|\overrightarrow{c^{\prime}}\right\|^{2}=1
$$

and, since $\overrightarrow{c^{\prime}}$ and $\vec{c}$ are orthogonal to each other we further have

$$
\left\|\overrightarrow{c^{\prime \prime}}\right\|^{2}=\|\vec{c}\|^{2}+\left.|D|^{2}\left\|\overrightarrow{c^{\prime}}\right\|\right|^{2}=1
$$

The value of $\|\vec{c}\|^{2}$ is fixed by the function $\Psi(x)$, in our case $\|\vec{c}\|^{2}=1$. Thus, the only possible solution to the above equation is $D=0$. This leads to the conclusion that amongst all the possible superposition giving rise to the same function $\Psi(x)$ on the interval, there is only one which is consistent with the physical significance of quantum mechanics.

\section{Conclusions}

An interesting result, arising by limiting the domain of a normalised function which is the superposition of the harmonic oscillator eigenfunctions, has been discussed. It was shown that, 
when restricted to a finite interval, such a function can be realized in many different ways. Although the mean values of the physical quantities are not affected in any significant manner, they can be the result of infinitely many different combinations of eigenfunctions. Uniqueness is restored by endowing the function with the significance of a wavepacket. It was proved that, in that case, there is only one set of coefficients that can fulfil the normalisation condition.
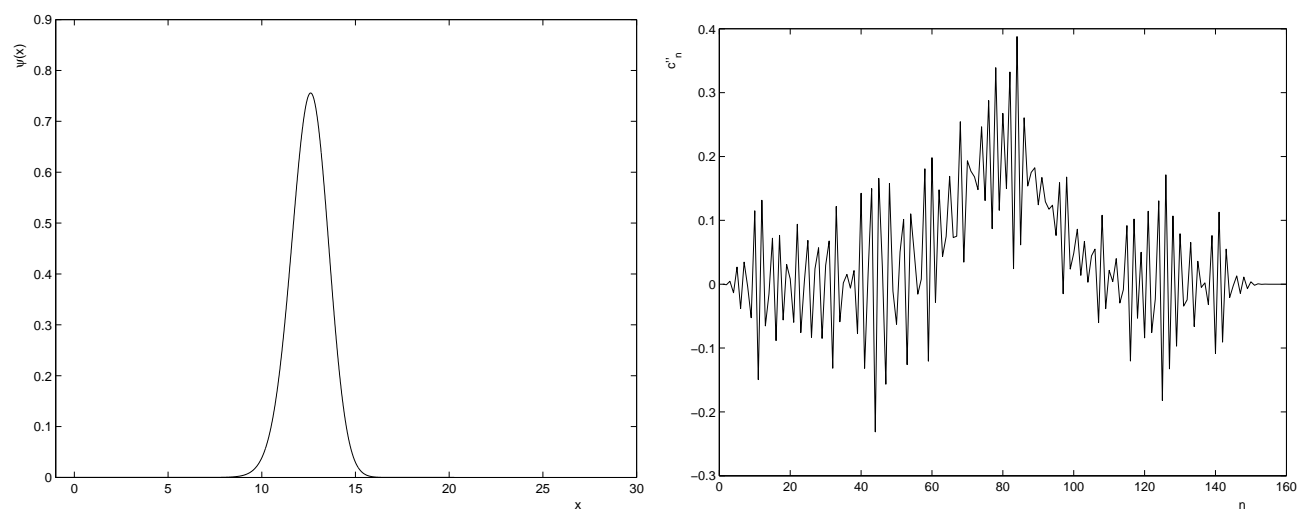

Figure 1: The graph on the left represents the function $\Psi$ constructed by using the coefficients given in (13) and also the coefficients $c_{n}^{\prime \prime}$ plotted in the right graph.
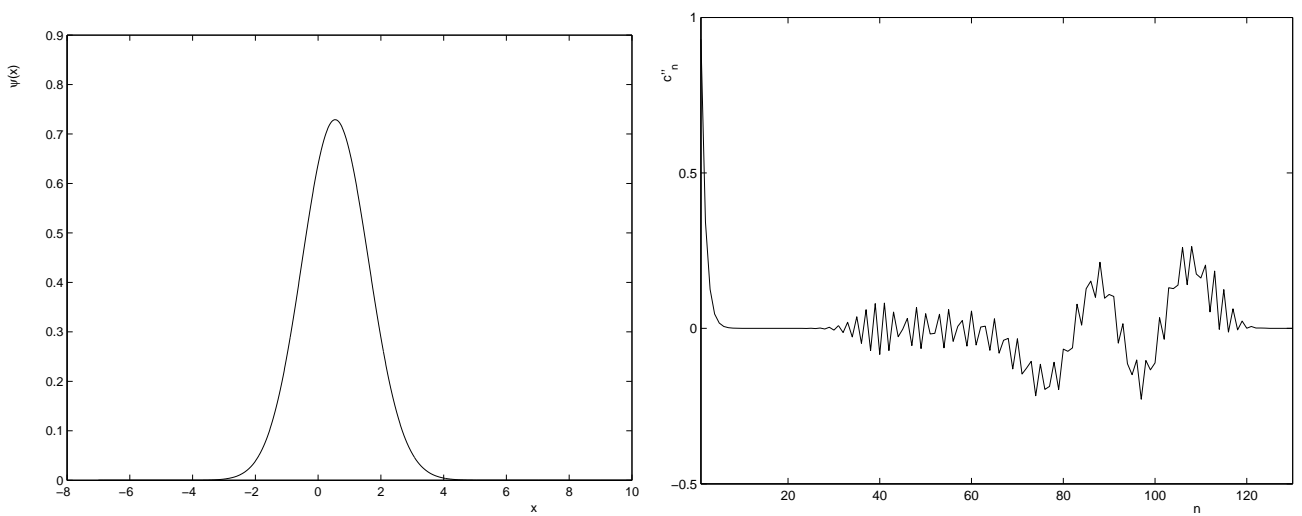

Figure 2: The graph on the left represents the function $\Psi$ constructed by using the coefficients given in (18) and also the coefficients $c_{n}^{\prime \prime}$ plotted in the right graph. 


\section{References}

[1] R. J. Duffin, A. C. Shaffer, "A Class of Nonharmonic Fourier Series," Trans. Amer. Math. Soc. 72, 341-366 (1952).

[2] I. Daubechies, A. Grossmann, Y. Meyer, "Painless nonorthogonal expansions", Journal of Mathematical Physics, 27, 1271-1283 (1986).

[3] C. Heil, D. Walnut, "Continuous and discrete wavelet transforms," SIAM Rev. 31, 628-666 (1989).

[4] G. Kaiser, Quantum Physics, Relativity and Complex Space time: Towards a New Synthesis (North-Holland, Amsterdam, 1990).

[5] S. T. Ali, J. P. Antoine, J. P. Gazeau, "Square integrability of group representation on homogeneous spaces. II. Coherent and quasi-coherent states. The case of the Poincaré group," Ann. Inst. Henri Poincaré 55, 857-890 (1991).

[6] S. T. Ali, J. P. Antoine, J. P. Gazeau, "Continuous Frames in Hilbert Space," Annals of Physics 222, 1-37 (1993).

[7] I. Daubechies, "The Wavelets Transform, Time Frequency Localization and Signal Analysis," IEEE Trans. Inform. Theory 36, 961-1005 (1990).

[8] D. Han, D. R. Larson, Frames, Bases and Group Representations, (Memoirs of the American Mathematical Society, Number 697, 2000)

[9] S. T. Ali, J. P. Antoine, J. P. Gazeau, "Coherent States, Wavelets and their generalizations", (Springer, London, 2000).

[10] L. Rebollo-Neira, A. Plastino, "The cross Wigner distribution as a generator of frames on the the Euclidean plane from frames on the real line", Journal of Physics A: Mathematical and General, $\operatorname{Vol}(33,15), 3053-3061,(2000)$. 
[11] L. Rebollo-Neira, "Frames in two dimensions arising from wavelet transforms", Proceedings of the Royal Society, series A, 457, 2013, 2079-2091. (2001).

[12] R. M. Young, An introduction to Nonharmonic Fourier Series (Academic Press, New York, 1980).

[13] K. Gröchening, Foundations of time-frequency analysis, (Birkhäuser, Boston, 2000)

[14] O. Christensen, An Introduction to Frames and Riesz Bases, (Birkhäuser, Berlin, 2002) 